\title{
Stem Cells for the Regeneration of Tendon and Ligament: A Perspective
}

\author{
Gun-Il Im, Tae-Kyung Kim \\ Research Institute for Integrative Regenerative Biomedical Engineering, Dongguk University, Goyang, Korea
}

Tendons are structures that connect muscles to the bones in our body and transmit the force generated by contraction of the muscles to the bones. Ligaments are structures that connect bones to bones, with histological properties similar to tendons. In tendon and ligament tissue, there are very small amounts of cells similar to mesenchymal stem cells (MSCs) called tendon stem/progenitor cells (TSPCs), or tenogenic stem cells. While the role of specific growth factors and transcription factors is well established in the osteogenic and chondrogenic differentiation of stem cells, a consensus has not been established for tenogenic differentiation. Injuries to tendons and ligaments are very common, but natural healing is very slow and inefficient due to limited vascularization. Currently, there is no adequate method for restoring extensive tendon or ligament defects. Procedures addressing the unmet need for regeneration of these tissues are needed. In this review, the current knowledge, as well as the authors' ideas and perspective on stem cell and regenerative medicine for tendon and ligament defects are presented.

Keywords: Tendon stem/progenitor cells, Osteogenesis, Tenogenesis, Ligaments, Tendons

\section{Structure and Function of Tendon and Ligament}

Tendons are structures connecting muscles to the bones in our body and transmit the force generated by contraction of the muscles to the bones. Ligaments connect bones to bones, with histological properties similar to tendons. Both tendons and ligaments can preserve elastic energy and withstand high tension (Fig. 1).

In the microstructure of the tendon, three tropocollagen

Received: May 28, 2020, Revised: July 12, 2020,

Accepted: August 16, 2020, Published online: October 31, 2020

Correspondence to Gun-Il Im

Research Institute for Integrative Regenerative Biomedical Engineering, Dongguk University, 27 Dongguk-ro, Ilsandong-gu, Goyang 10326, Korea

Tel: +82-31-961-7315, Fax: +82-31-961-7314

E-mail: gunil@duih.org

(a) This is an open-access article distributed under the terms of the Creative Commons Attribution Non-Commercial License (http://creativecommons.org/ licenses/by-nc/4.0/), which permits unrestricted non-commercial use, distribution, and reproduction in any medium, provided the original work is properly cited.

Copyright (C) 2020 by the Korean Society for Stem Cell Research chains are intertwined with one another to form microfibrils. The microfibrils are stacked in parallel to form fibrils, and the fibrils gather to form fascicles that are wrapped by endotenon. The fascicles gather to form tendons, which are surrounded by epitenon. Epitenon is wrapped by paratenon, which contains blood vessels and nerves that feed the tendon (Fig. 2).

Collagen primarily provides resistance to tensile forces. While type 1 collagen comprises $95 \%$ of the whole collagen, there are also types 3, 5, 6, 12, 14, and 15 collagen. Type 3 collagen is the most common type of collagen in tendons after type 1 collagen. Type 3 collagen tends to in-
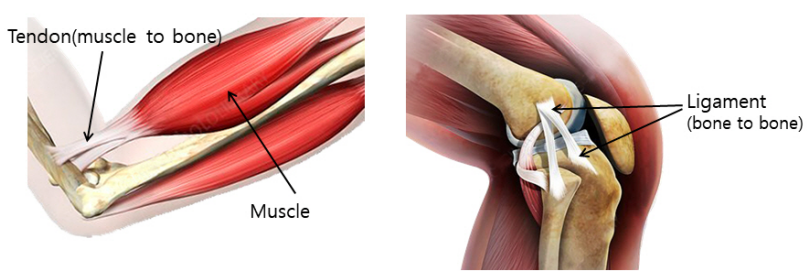

Fig. 1. Anatomy and function of tendons and ligaments. 


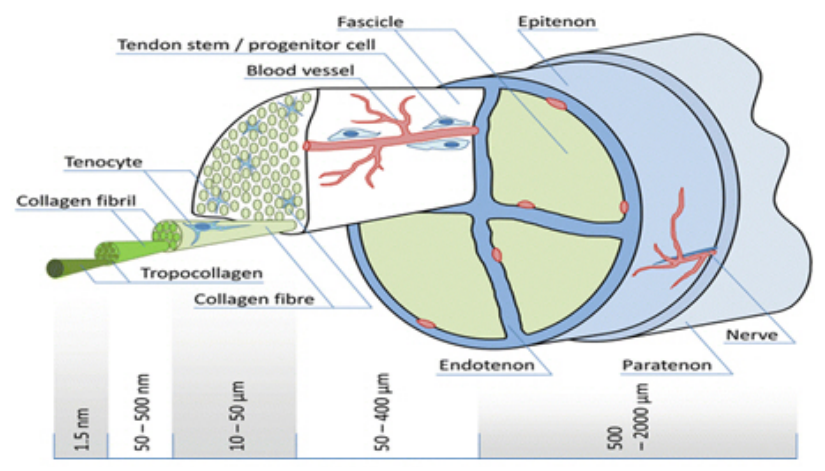

Fig. 2. Microstructure of a tendon.

crease in the course of tendinitis or tendon healing. Elastin endows an elastic property to tendons. Type 1 collagen accounts for $65 \sim 80 \%$ of the dry weight of tendons, while elastin contributes 2\%. Extracellular matrix (ECM) provides lubrication and viscoelasticity to the tendon and maintains the fibrous structure. The ECM of tendon is comprised of glycoproteins, including tenascin, decorin (DCN), biglycan (BGN), fibromodulin (FMOD), and lumican (LUM).

Tenomodulin (Tnmd) is a type 2 transmembrane glycoprotein specific to the tendon that plays an important role in cell proliferation and tendon maturation. Tnmd is regulated by scleraxis (SCX), a tendon-specific transcription factor. When Tnmd is deficient, the proliferative capacity of tendon progenitor cells decreases, and aging progresses rapidly. As it is not found in other musculoskeletal cells, Tnmd is a good marker of tenogenic differentiation.

In normal tendons, the fibers are parallel and there are few cells. In tendinosis, the number of cells increases with the proliferation of blood vessels and mucus degeneration of the tendon substance (1-3).

\section{Tendon/Ligament-Specific Stem Cells}

In tendon and ligament tissue, there are very small numbers of cells similar to mesenchymal stem cells (MSCs), called tendon stem/progenitor cells (TSPCs) or tenogenic stem cells $(4,5)$. TSPCs have all the properties of general MSCs, such as the presence of specific surface markers, self-replication ability, and the ability to differentiate into bone, cartilage, and fat. Unlike bone marrow stem cells (BMSCs) or adipose stem cells (ASCs), SCX is always expressed in TSPCs. When implanted, TSPCs form ectopic tissue. When SCX is introduced into bone marrow stem cells, these cells acquire the characteristics of TSPCs. The expression of SRY-Box transcription factor 9 (SOX9), a marker of cartilage formation, as well as osteogenic po-

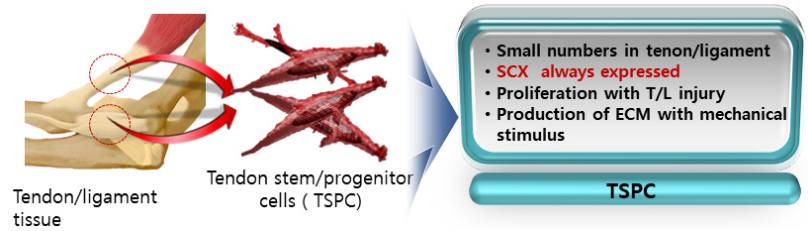

Fig. 3. Characteristics of tendon stem/progenitor cells (TSPCs).

tential, is significantly reduced in these cells (6). In TSPCs, biglycan and fibromodulin proteins in the ECM of tendon maintain the microenvironment (stem cell niche) and control bone morphogenetic protein (BMP) signals to prevent osteogenic differentiation $(4,5)$. While TSPCs do not proliferate under normal conditions, they generate ECM under dynamic stimulation (Fig. 3) (4, 5).

\section{Transcription Factors Involved in the Differentiation of Tendon}

While the role of specific growth factors and transcription factors is well established in the osteogenic and chondrogenic differentiation of stem cells, surprisingly, a consensus has not been established for tenogenic differentiation $(1,7)$. The currently known transcription factors necessary for tendon development include SCX, Mohawk $(\mathrm{MKX})$, and early response protein (Egrl). SCX is a helix-loop-helix transcription factor, first known as a tendon/ligament specific factor, expressed in the early stages of tendon development. It promotes the transcription of type 1 collagen a1/a2, aggrecan, and Tnmd genes. When SCX is knocked out, tendon development is suppressed due to the absent aggregation of tendon progenitor cells, resulting in the disorderly arrangement of fibrils. The development of bone tubercles to which ligaments are attached is also inhibited. The expression of SCX in ectopic embryonic stem cells initiates tenogenic differentiation. SCX increases the production of ECM but does not mature tendon structures $(1,7)$.

MKX also regulates the expression of type 1 collagen in tendon development. The deficient expression of MKX does not change the number of cells, but it reduces the size of the tendon and the diameter of the collagen fibers. MKX promotes the expression of SCX by binding to the transforming growth factor (TGF)- $\beta 2$ promoter. MKX inhibits osteogenic, chondrogenic, and adipogenic differentiation, as well as the proliferative potential of MSCs. MKX plays a more important role in the maturation and maintenance of tendons, whereas SCX initiates tenogenic differentiation (7-9). A reduction in MKX expression was reported in the anterior cruciate ligament (ACL) of pa- 
tients with tendinopathies or knee osteoarthritis. MKX also inhibits chondrogenic metaplasia of mature tendons by inhibiting SOX9 $(7,9)$.

Egrl is a zinc finger transcription factor, which targets the expression of the TGF- $\beta 2$ promoter. Egrl knockout suppresses the production of SCX and type 1 collagen al/a2, thus reducing the tensile strength of the tendon (Fig. 4) (1, 7).

\section{Growth Factors that Induce Tendon Proliferation and Differentiation}

Growth factors that were investigated for TSPC proliferation are connective tissue growth factor (CTGF), insulin-like growth factor $-1 / 2$, platelet-derived growth factor $\mathrm{BB}$ (PDGF-BB), and basic fibroblast growth factor (bFGF). Connective tissue growth factor (CTGF/CCN2) increases the expression of type 1 collagen and tenascin. It also promotes the production of SCX and tenomodulin by modulating FAK and ERK1/2 signaling (10). CTGF supplementation enriched a CD146 (tendon stem cell marker) + TSPC subpopulation, and induced proliferation and tenogenic differentiation from rat patellar tendon-derived cells (11). CTGF also recruited CD146+ cells and enhanced tendon healing in vivo (11). While either PDGF$\mathrm{BB}$ or bFGF significantly increased cell proliferation and matrix synthesis in tendon fibroblast culture $(12,13)$, co-treatment of PDGF-BB combined with bFGF dose-dependently led to an accelerated cell proliferation (13). When combinations of growth factors are utilized, unexpected interactions can be engendered with activation of different signaling pathways. Of several different combina- tions, IGF1 plus GDF5 demonstrated the most spectacular increase in the expression of tenogenic marker and collagen synthesis (14).

TGF- $\beta$ signaling pathway is involved in inducing and maintaining tendon progenitors in the developing embryo: disturbance in either ligands or receptors resulting in loss of all tendon structure $(14,15)$. TGF- $\beta$ ligands are potent inducers of tendon markers including $\operatorname{SCX}(14,15)$. Myostatin, another TGF- $\beta$ Smad2/3 subfamily member, also has similar tenogenic properties (16). While several BMPs inhibit tenogenic induction, a subset of BMPs positively regulate tendon-specific differentiation (17-19). Growth differentiation factors (GDF)-5,6,7 relatively specific growth factors for tendon differentiation, also called bone morphogenetic protein (BMP) -14,13,12 or cartilage-derived morphogenetic protein (CDMP) -1,2,3 respectively, are closely related factors belonging to the TGF- $\beta$ superfamily (20-23). The treatment of MSCs with these factors was shown to promote ectopic tendon/ligament differentiation, whereas other BMPs promoted osteogenic or chondrogenic differentiation (23). GDF factors induce tenogenic differentiation through Smad8 signaling system. Without these GDF factors, the size of collagen fiber decreases, and healing is delayed in the case of tendon injury. A deficiency in one factor can be compensated by other factors. Sutures coated with GDF5/BMP14 were reported to promote collagen synthesis. When treated with GDF5/BMP14, rat TSPCs had increased SCX expression with reduced adipogenic and chondrogenic potential (24). GDF5/BMP14 treatment also increased cell proliferation and matrix content of murine tendon fibroblasts $(25,26)$. Collagen sponges containing GDF7/BMP12 promoted the

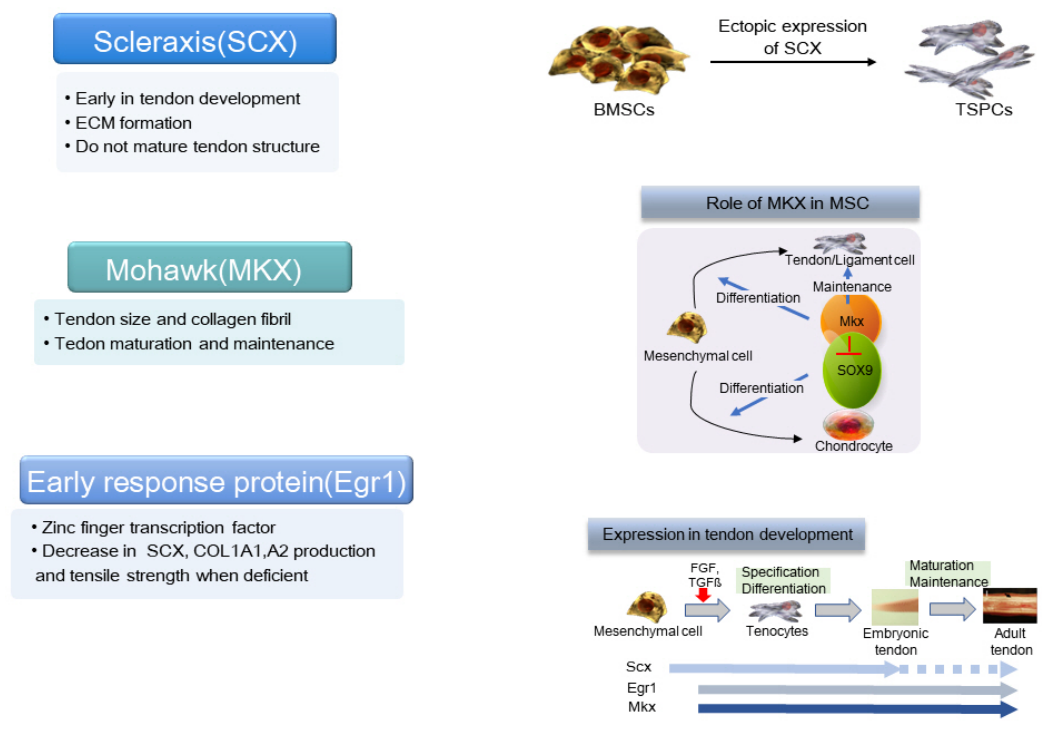

Fig. 4. Transcription factors related to tendon development. 
healing of tendon damage in patients with rotator cuff injuries in clinical trials (14). Similarly, GDF7/BMP12 upregulated tendon markers SCX, Tnmd, type 1 collagen and tenascin in rat TSPCs (27). BMP12 combined with CTGF overexpression had an additive effect on tenogenic gene expressions (27). TSPCs co-transfected with BMP12 and CTGF genes had increased SCX, type 1 collagen a1, type 3 collagen al, and tenascin expression while osteo-, chondro-, and adipogenic markers were inhibited (28).

\section{Mechanical and Environmental Factors for Tenogenic Differentiation}

Another strategy to induce tenogenic differentiation is mechanical stimulations similar to those seen in native microenvironments $(29,30)$. Although individual tendons undergo specific loading subject to different anatomic locations, the general loading type for most tendons is uniaxial tension. When uniaxial and biaxial stimulations were directly compared, uniaxial loading was more strongly tenogenic to TSPCs than biaxial loading, which induced a mixed osteogenic, adipogenic, and chondrogenic signals (30). Uniaxial loading of three-dimensional TSPC significantly promoted tenogenic differentiation, neo-tendon formation, and mechanical properties of formed tendon (30).

TSPCs typically show reduced proliferation and ECM production as well as decreased tendon and stemness markers at later passages of culture $(31,32)$. TSPCs cultured at low oxygen levels showed enhanced cell proliferation and increased gene expression of stemness and tendon markers while osteogenic, adipogenic, and chondrogenic gene expressions were reduced, avoiding problems seen in long-term culture (33-35).

\section{Induction of Tenogenic Differentiation from Pluripotent Stem Cells}

Tenogenic stem cells can be induced from pluripotent stem cells. Komura et al. (36) developed a protocol for inducing tenogenic differentiation from mouse induced pluripotent stem cells (miPSCs) using serial treatment of Wnt3a and activin A, basic FGF, and TGF- $\beta 1$ and bFGF. They created reporter mice that express enhanced green fluorescent protein (EGFP) driven by SCX promoter and generated iPSC from these mice. The iPSC-derived EGFPpositive cells exhibited elevated expression of tendon-specific genes, including SCX, MHK, Tnmd, and FMOD, indicating that they have tenocyte-like properties.

McClellan et al. (37) differentiated equine embryonic stem cells (eESCs) to tenocytes by culturing without feeders in ESC media lacking LIF and in the presence of 20 $\mathrm{ng} / \mathrm{ml}$ TGF- $\beta 3$ for 14 days. Dale et al. (38) induced tenogenic differentiation of hESCs using BMP12 and BMP13 in the presence of ascorbic acid (AA). A tenocyte-like elongated morphology was observed in hESC after 40-days continuous supplementation with BMP12, BMP13 and AA.

\section{Current Status of Treatment for Tendon and Ligament Injuries}

Tendon and ligament damage is very common, with about 3 5 million cases worldwide each year, accounting for $50 \%$ of the physical injuries (39). Tendon and ligament injuries are mostly caused by degenerative changes due to overuse, aging, or sports injuries. Natural healing is very slow and inefficient due to the low vascularization of tendons and ligaments $(1,4,5)$. When a tendon ruptures, it undergoes a healing process that consists of inflammatory, proliferative, matrix formation, and remodeling phases. While surgical repair is usually performed, re-rupture frequently occurs due to incomplete tendon regeneration and it is exceedingly difficult to regain pre-rupture strength.

ACL rupture is one of the most common sports injuries. It occurs frequently at 1 in 3,000 people, with 200,000 surgical procedures performed every year in the United States alone (40). ACL rupture is currently treated with reconstructive procedures using autologous or allograft grafts. Complications, such as rupture, donor site morbidity, instability, and secondary osteoarthritis, are problems after ACL rupture and repair.

Rotator cuff tears (RCTs), the most common degenerative tendon disease, occur in about $30 \%$ of people over the age of 60 . Of them, $35 \%$ progress to the extent that daily life is difficult (41, 42). Around 200,000 operations are performed for RCTs each year in the United States, costing about 500 million dollars (43). Unfortunately, the re-rupture of large size RCTs after surgical repair is quite common, leading to treatment failure. Regenerative treatment has been considered or applied, including growth factors, biomaterials, platelet-rich plasma, and cell therapy $(1,5)$.

\section{Need for Regenerative Treatment for Tendon and Ligament Injuries}

As a regenerative cell therapy for tendon and ligament regeneration, autologous bone marrow aspirate concen- 


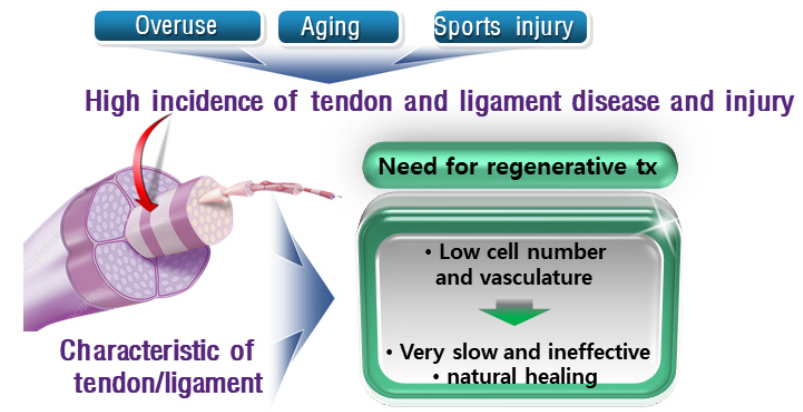

Fig. 5. Need for regenerative therapy for tendon and ligament regeneration.

trate, autologous stromal vascular fraction or platelet-rich plasma have been applied. It should be noted that they are not specific treatments for tendon regeneration. Also, there are great differences in the method of administration depending on the treating physicians, with controversial effects.

There is currently no adequate method of treatment for extensive tendon defects seen in RCTs. A treatment is necessary that can respond to the unmet need to augment tendon suturing in wide tendon defects with additional regenerative effects. In ACL rupture, as a direct repair is mostly unsuccessful, reconstruction using autogenous or allogeneic tendon is currently the standard treatment, which is a larger operation than primary suture. Also, autografting is associated with donor site morbidity while the latter has an associated re-rupture rate. Methods are necessary that can reduce treatment morbidity by increasing the success rate of tendon suturing and reduce the re-rupture rate of allograft reconstruction. In the case of tendinopathies, a successful treatment method is needed to induce regeneration of the degenerative tendon causing discomfort and deterioration in the quality of life (Fig. 5).

There are sporadic reports on the clinical application of stem cell for tendon regeneration. Hernigou et al. (44) evaluated the efficiency of biologic augmentation of rotator cuff repair with iliac crest BMSCs. Forty-five patients in the study group received concentrated BMSCs $(51,000 \pm$ 25,000 ) as an adjunct to rotator cuff repair at the time of arthroscopy. BMSC injection during rotator cuff repair enhanced the healing rate and improved the quality of repair. At the most recent follow-up of 10 years, intact rotator cuffs were found in $39(87 \%)$ of the 45 patients in the MSC-treated group, but just 20 (44\%) of the 45 patients in the control group (44).

Jo et al. (45) assessed the result of intratendinous injection of ASCs for partial-thickness rotator cuff tear at mid-term follow-up. The first part of the study consisted of 3 dose-escalation groups, with 3 patients each: low-dose $\left(1.0 \times 10^{7}\right.$ cells $)$, mid-dose $\left(5.0 \times 10^{7}\right)$, and high-dose $(1.0 \times$ $10^{8}$ ) groups. For the second part, 10 patients with high dose treatment were added. Intratendinous injection of ASCs reduced shoulder pain by approximately $90 \%$ at 1 and 2 years in the mid- and high-dose groups. The strength of rotator cuff muscles significantly increased by greater than $50 \%$ at 2 years in the high-dose group. Magnetic resonance imaging showed that the volume of tendon defects in the high-dose group nearly disappeared at 1 year and did not recur at up to 2 years (45).

\section{Future Strategy: Generation of Tendon and Ligament-Specific Stem Cells by Gene Transfer of Tendon-Specific Transcription Factor}

Considering that the number of TSPS available from tendon harvest is limited, a viable strategy would be to derive therapeutic tenogenic stem cells from BMSCs or ASCs. While it is possible to induce MSCs into tenogenic stem cells by incubating them with growth factors such as GDFs, the in vitro culture time is longer, and the cost of serum and growth factors required for culture is increased accordingly. In comparison, if tendon-differentiated transcription factor genes can be transferred to MSCs with high efficiency, these MSCs are expected to have the properties of tenogenic stem cells. It has already been confirmed that BMSCs and ASCs, which have SCX and $\mathrm{MHK}$ genes transferred using adenovirus, have properties similar to TSPCs $(1,6,8)$. MSCs to which the gene for differentiation transcription factor has been transferred can be used as cell therapy for tendon and ligament regeneration without separation or additional culture. Therefore, it is expected that the gene transfer of tendon-specific transcription factors will be effective in generating tendon and ligament-specific stem cells. Use of nonviral vectors or adeno-associated viral vector should be considered because of nonlethal nature of the tendon or ligament disease. Combining the application of these tenogenic stem cells with current surgical repair techniques may provide a less expensive and technically feasible regenerative technology for tendon and ligament injuries resistant to current treatment.

\section{Acknowledgments}

This review was supported by a grant of the National Research Foundation of Korea (NRF-2020R1A2C2008266). 


\section{Potential Conflict of Interest}

The authors have no conflicting financial interest.

\section{References}

1. Docheva D, Müller SA, Majewski M, Evans CH. Biologics for tendon repair. Adv Drug Deliv Rev 2015;84:222-239

2. Alberton P, Dex S, Popov C, Shukunami C, Schieker M, Docheva D. Loss of tenomodulin results in reduced self-renewal and augmented senescence of tendon stem/progenitor cells. Stem Cells Dev 2015;24:597-609

3. Figueroa D, Figueroa F, Calvo R. Patellar tendinopathy: diagnosis and treatment. J Am Acad Orthop Surg 2016;24: e184-e192

4. Huang $\mathrm{AH}, \mathrm{Lu} \mathrm{HH}$, Schweitzer R. Molecular regulation of tendon cell fate during development. J Orthop Res 2015;33: 800-812

5. Walia B, Huang AH. Tendon stem progenitor cells: understanding the biology to inform therapeutic strategies for tendon repair. J Orthop Res 2019;37:1270-1280

6. Alberton P, Popov C, Prägert M, Kohler J, Shukunami C, Schieker M, Docheva D. Conversion of human bone marrow-derived mesenchymal stem cells into tendon progenitor cells by ectopic expression of scleraxis. Stem Cells Dev 2012;21:846-858

7. Asahara $\mathrm{H}$, Inui $\mathrm{M}$, Lotz $\mathrm{MK}$. Tendons and ligaments: connecting developmental biology to musculoskeletal disease pathogenesis. J Bone Miner Res 2017;32:1773-1782

8. Otabe K, Nakahara H, Hasegawa A, Matsukawa T, Ayabe F, Onizuka N, Inui M, Takada S, Ito Y, Sekiya I, Muneta T, Lotz M, Asahara H. Transcription factor Mohawk controls tenogenic differentiation of bone marrow mesenchymal stem cells in vitro and in vivo. J Orthop Res 2015;33: $1-8$

9. Liu H, Zhang C, Zhu S, Lu P, Zhu T, Gong X, Zhang Z, Hu J, Yin Z, Heng BC, Chen X, Ouyang HW. Mohawk promotes the tenogenesis of mesenchymal stem cells through activation of the TGF $\beta$ signaling pathway. Stem Cells 2015;33:443-455

10. Li X, Pongkitwitoon S, Lu H, Lee C, Gelberman R, Thomopoulos S. CTGF induces tenogenic differentiation and proliferation of adipose-derived stromal cells. J Orthop Res 2019;37:574-582

11. Lee CH, Lee FY, Tarafder S, Kao K, Jun Y, Yang G, Mao JJ. Harnessing endogenous stem/progenitor cells for tendon regeneration. J Clin Invest 2015;125:2690-2701

12. Yoshikawa Y, Abrahamsson SO. Dose-related cellular effects of platelet-derived growth factor-BB differ in various types of rabbit tendons in vitro. Acta Orthop Scand 2001; 72:287-292

13. Thomopoulos S, Harwood FL, Silva MJ, Amiel D, Gelberman RH. Effect of several growth factors on canine flexor tendon fibroblast proliferation and collagen synthesis in vitro. J Hand Surg Am 2005;30:441-447

14. Pryce BA, Watson SS, Murchison ND, Staverosky JA,
Dünker N, Schweitzer R. Recruitment and maintenance of tendon progenitors by TGFbeta signaling are essential for tendon formation. Development 2009;136:1351-1361

15. Havis E, Bonnin MA, Olivera-Martinez I, Nazaret N, Ruggiu M, Weibel J, Durand C, Guerquin MJ, BonodBidaud C, Ruggiero F, Schweitzer R, Duprez D. Transcriptomic analysis of mouse limb tendon cells during development. Development 2014;141:3683-3696

16. Mendias CL, Bakhurin KI, Faulkner JA. Tendons of myostatin-deficient mice are small, brittle, and hypocellular. Proc Natl Acad Sci U S A 2008;105:388-393

17. Schweitzer R, Chyung JH, Murtaugh LC, Brent AE, Rosen V, Olson EN, Lassar A, Tabin CJ. Analysis of the tendon cell fate using Scleraxis, a specific marker for tendons and ligaments. Development 2001;128:3855-3866

18. Bénazet JD, Pignatti E, Nugent A, Unal E, Laurent F, Zeller R. Smad4 is required to induce digit ray primordia and to initiate the aggregation and differentiation of chondrogenic progenitors in mouse limb buds. Development 2012;139:4250-4260

19. Rui YF, Lui PP, Wong YM, Tan Q, Chan KM. BMP-2 stimulated non-tenogenic differentiation and promoted proteoglycan deposition of tendon-derived stem cells (TDSCs) in vitro. J Orthop Res 2013;31:746-753

20. Prabhath A, Vernekar VN, Sanchez E, Laurencin CT. Growth factor delivery strategies for rotator cuff repair and regeneration. Int J Pharm 2018;544:358-371

21. Haddad-Weber M, Prager P, Kunz M, Seefried L, Jakob F, Murray MM, Evans CH, Nöth U, Steinert AF. BMP12 and BMP13 gene transfer induce ligamentogenic differentiation in mesenchymal progenitor and anterior cruciate ligament cells. Cytotherapy 2010;12:505-513

22. Wang $\mathrm{D}$, Jiang $\mathrm{X}, \mathrm{Lu} \mathrm{A}, \mathrm{Tu} M$, Huang W, Huang $\mathrm{P}$. BMP14 induces tenogenic differentiation of bone marrow mesenchymal stem cells in vitro. Exp Ther Med 2018;16: 1165-1174

23. Zhang YJ, Chen X, Li G, Chan KM, Heng BC, Yin Z, Ouyang HW. Concise review: stem cell fate guided by bioactive molecules for tendon regeneration. Stem Cells Transl Med 2018;7:404-414

24. Holladay C, Abbah SA, O'Dowd C, Pandit A, Zeugolis DI. Preferential tendon stem cell response to growth factor supplementation. J Tissue Eng Regen Med 2016;10:783-798

25. Keller TC, Hogan MV, Kesturu G, James R, Balian G, Chhabra AB. Growth/differentiation factor-5 modulates the synthesis and expression of extracellular matrix and celladhesion-related molecules of rat Achilles tendon fibroblasts. Connect Tissue Res 2011;52:353-364

26. Hogan M, Girish K, James R, Balian G, Hurwitz S, Chhabra AB. Growth differentiation factor-5 regulation of extracellular matrix gene expression in murine tendon fibroblasts. J Tissue Eng Regen Med 2011;5:191-200

27. Liu J, Tao X, Chen L, Han W, Zhou Y, Tang K. CTGF positively regulates BMP12 induced tenogenic differentiation of tendon stem cells and signaling. Cell Physiol Biochem 2015;35:1831-1845 
28. Xu K, Sun Y, Kh Al-Ani M, Wang C, Sha Y, Sung KP, Dong N, Qiu X, Yang L. Synergistic promoting effects of bone morphogenetic protein 12 /connective tissue growth factor on functional differentiation of tendon derived stem cells and patellar tendon window defect regeneration. J Biomech 2018;66:95-102

29. Popov C, Burggraf M, Kreja L, Ignatius A, Schieker M, Docheva D. Mechanical stimulation of human tendon stem/progenitor cells results in upregulation of matrix proteins, integrins and MMPs, and activation of p38 and ERK1/2 kinases. BMC Mol Biol 2015;16:6

30. Wang T, Thien C, Wang C, Ni M, Gao J, Wang A, Jiang Q, Tuan RS, Zheng Q, Zheng MH. 3D uniaxial mechanical stimulation induces tenogenic differentiation of tendon-derived stem cells through a PI3K/AKT signaling pathway. FASEB J 2018;32:4804-4814

31. Yao L, Bestwick CS, Bestwick LA, Maffulli N, Aspden RM. Phenotypic drift in human tenocyte culture. Tissue Eng 2006;12:1843-1849

32. Tan Q, Lui PP, Rui YF. Effect of in vitro passaging on the stem cell-related properties of tendon-derived stem cells-implications in tissue engineering. Stem Cells Dev 2012;21:790-800

33. Lee WY, Lui PP, Rui YF. Hypoxia-mediated efficient expansion of human tendon-derived stem cells in vitro. Tissue Eng Part A 2012;18:484-498

34. Zhang J, Wang JH. Human tendon stem cells better maintain their stemness in hypoxic culture conditions. PLoS One 2013;8:e61424

35. Yu Y, Lin L, Zhou Y, Lu X, Shao X, Lin C, Yu K, Zhang $\mathrm{X}$, Hong J, Chen Y. Effect of hypoxia on self-renewal capacity and differentiation in human tendon-derived stem cells. Med Sci Monit 2017;23:1334-1339

36. Komura S, Satake T, Goto A, Aoki H, Shibata H, Ito K, Hirakawa A, Yamada Y, Akiyama H. Induced pluripotent stem cell-derived tenocyte-like cells promote the regeneration of injured tendons in mice. Sci Rep 2020;10:3992

37. McClellan A, Paterson YZ, Paillot R, Guest DJ. Equine fe- tal, adult, and embryonic stem cell-derived tenocytes are all immune privileged but exhibit different immune suppressive properties in vitro. Stem Cells Dev 2019;28:14131423

38. Dale TP, Mazher S, Webb WR, Zhou J, Maffulli N, Chen GQ, El Haj AJ, Forsyth NR. Tenogenic differentiation of human embryonic stem cells. Tissue Eng Part A 2018;24: 361-368

39. Ljungqvist A, Schwellnus MP, Bachl N, Collins M, Cook J, Khan KM, Maffulli N, Pitsiladis Y, Riley G, Golspink G, Venter D, Derman EW, Engebretsen L, Volpi P. International Olympic Committee consensus statement: molecular basis of connective tissue and muscle injuries in sport. Clin Sports Med 2008;27:231-239

40. Singh N. International epidemiology of anterior cruciate ligament injuries. Orthop Res Online J 2017;1:94-96

41. Khatri C, Ahmed I, Parsons H, Smith NA, Lawrence TM, Modi CS, Drew SJ, Bhabra G, Parsons NR, Underwood M, Metcalfe AJ. The natural history of full-thickness rotator cuff tears in randomized controlled trials: a systematic review and meta-analysis. Am J Sports Med 2019;47:1734-1743

42. Reilly P, Macleod I, Macfarlane R, Windley J, Emery RJ. Dead men and radiologists don't lie: a review of cadaveric and radiological studies of rotator cuff tear prevalence. Ann R Coll Surg Engl 2006;88:116-121

43. Chalmers PN, Ross H, Granger E, Presson AP, Zhang C, Tashiian RZ. The effect of rotator cuff repair on natural history: a systematic review of intermediate to long-term outcomes. JB JS Open Access 2018;3:e0043

44. Hernigou P, Flouzat Lachaniette CH, Delambre J, Zilber S, Duffiet P, Chevallier N, Rouard H. Biologic augmentation of rotator cuff repair with mesenchymal stem cells during arthroscopy improves healing and prevents further tears: a case-controlled study. Int Orthop 2014;38:1811-1818

45. Jo CH, Chai JW, Jeong EC, Oh S, Yoon KS. Intratendinous injection of mesenchymal stem cells for the treatment of rotator cuff disease: a 2-year follow-up study. Arthroscopy 2020;36:971-980 